\title{
Association of Hepatitis ' $E$ ' Virus With an Outbreak of Hepatitis at a Military Training Camp in Nepal
}

\author{
Edward T. Clayson, ${ }^{3}$ David W. Vaughn, ${ }^{1}$ Bruce L. Innis, ${ }^{2}$ Mrigendra P. Shrestha, ${ }^{1}$ Rajiv
Pandey, and Devendra B. Malla ${ }^{3}$ \\ ${ }^{1}$ Department of Virology, Armed Forces Research Institute of Medical Sciences, Bangkok, Thailand \\ ${ }^{2}$ Department of Virus Diseases, Walter Reed Army Institute of Research, Washington D.C. \\ ${ }^{3}$ Birendra Army Hospital, Kathmandu, Nepal.
}

From 29 January 1995 to 15 March 1995, an outbreak of hepatitis occurred among 692 soldiers at an isolated training camp $25 \mathrm{~km}$ east of Kathmandu. Thirty-two cases occurred approximately 8 weeks after arrival of soldiers at the camp. To determine the etiology of the outbreak, patient sera were examined for evidence of infection with hepatitis $\mathrm{A}, \mathrm{B}, \mathrm{C}$, and $\mathrm{E}$ viruses using commercially available enzyme-linked immunosorbent assay (ELISA) kits. The polymerase chain reaction (PCR) was used to detect hepatitis E virus (HEV) RNA. Evidence of recent infection (IgM to HEV and / or HEV RNA) was found in all but two patients, whereas none had evidence of recent infection with hepatitis $\mathrm{A}, \mathrm{B}$ or $\mathrm{C}$ viruses. Therefore, the outbreak was attributed to HEV. Fecally contaminated drinking water was suspected as the source of the outbreak. To determine the extent of HEV infections among those without clinical hepatitis, sera from the remaining soldiers were examined for markers of HEV infection. Evidence of past infection ( $\mathrm{IgG}$ to HEV in the absence of IgM or HEV RNA) was found among 204 soldiers (Prevalence $=30 \%$ ), leaving 488 individuals susceptible to infection at the onset of the outbreak. Evidence of recent infection was found among another 83 individuals. We conclude that most exposed, susceptible soldiers sustained HEV infection without experiencing overt hepatitis. If the levels of virus inoculum and prior immunity in this population were typical, inapparent infection may be the usual adult response to virus exposure in an endemic area.

KEY WORDS: HEV; RT- PCR; viral hepatitis, epidemiology; adults.

\section{Introduction}

The hepatitis E virus (HEV) is a small, nonenveloped, positive strand RNA virus, and is the leading cause of enterically transmitted, non-A hepatitis worldwide. This virus is responsible for major outbreaks of acute hepatitis in developing countries, especially in tropical and subtropical regions of the world. Disease outbreaks are usually associated with fecally contaminated drinking water. In disease-endemic areas, $\mathrm{HEV}$ appears to be continuously present in the environment as evidenced by the finding of the virus in wild animals [Clayson et al., 1995 b] and in drinking water [Jothikumar et al., 1993, 1995]. Sporadic cases of HEV infection are common in endemic regions, but are rare in industrial countries where they tend to be imported from endemic regions.

Infection with $\mathrm{HEV}$ is believed to be self-limiting as no cases of chronic hepatitis $\mathrm{E}$ have been reported. The majority of $\mathrm{HEV}$ infections likely occur without illness 'Longer et all, 1996; Clayson et all, 1997]. When disease occurs, the average incubation period for HEV appears to be 40 days (range 15-16 days) and is slightly longer than the incubation period for hepatitis. A virus (HAV) [ Balayan, 1991]. The virus can often be found in the sera and feces of infected individuals [Clayson et al., 1995c]. Illness associated with HEV infection can range from moderate to severe icteric disease. Death due to fulminate hepatitis may result from $\mathrm{HEV}$ infection : the mortality rate In fan from approximately 1 to $2 \%$ among the general population to $20-30 \%$ in pregnant women. In nonfatal cases, recovery from illness is complete. Hepatitis $\mathrm{E}$ is a public health concern as vaccines are not available and immune serum globulin appears to have little to no protective effect [Joshi et all., 1985, Zhuang et al., 1991; Khuroo and Dar, 1992]. 


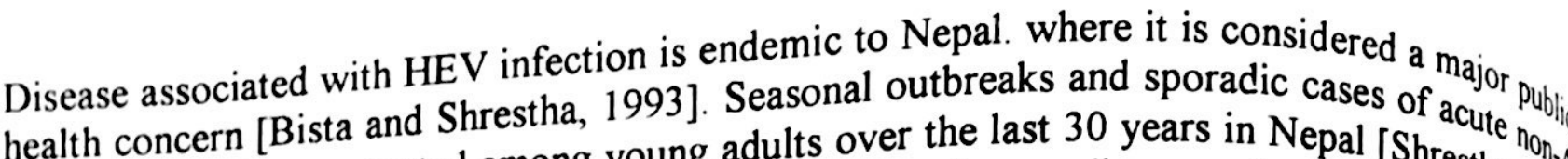
hepatitis have been reported among young adults over the last 30 years in Nepal [Shrestha, 198 . 1991; Kane et al., 1984]. Recently, these outbreaks and information is known about the epidemioled io HEV infection [Claysod to the Kathmandu Valley. The prevalence of IgG to HEV in Kathmy of appears to be age specific, with antibody prevalence increasing with age to a maximum of approximately $30 \%$ in adults 40 years or older [Longer et al., 1994, Clayson et al., 1997. $\mathrm{No}$ increase in antibody prevalence is apparente to be greatest among teenagers (45 and vinus transmission, disease and infection rates appear al., 1997]. Both disease and infection rates 99 per 1,000 person-years, respectively) [Chan 19 years. Therefore, disease and infection appeappes. to decline with age in persons older Information regarding infection and disease retear to be most prevalent among young adults. epidemic virus transmission is not available.

In 1995 beginning in late January and ending in mid-March, an outbreak of acute hepatitis occurred among military personnel at an isolated training camp located approximately $25 \mathrm{~km}$ east of Kathmandu. The outbreak was recognized early be the attending Nepalese military physician, who promptly warned the Nepalese Army Medical Department. An outbreak investigation was initiated to determine the cause of the outbreak and to take steps to limit the spread of the outbreak. In addition, the occurrence of an outbreak of hepatitis at an isolated location among a well-defined population provided a unique opportunity to gain epidemiologic information regarding the cause of the outbreak. This is a report of that outbreak investigation.

\section{Materials and Methods}

\section{Study Area}

The study area was an isolated military training camp approximately $25 \mathrm{~km}$ east of Kathmandu. At the time of the outbreak, the camp was occupied by 692 Nepalese soldiers who had recently arrived from all regions of Nepal to form and train a new unit for deployment. All soldiers were male. The camp lies in a region of rolling hills covered by forest. Open air structures and tents were used as training, dining, and sleeping areas and were generally located on higher ground Toilet facilities consisted of open air pit latrines and were located near training, dining and sleeping areas. Soldiers obtained drinking water from multiple natural outdoor sources (e.g, springs, creeks, etc.) generally located down hill from training, dining, and sleeping areas.

\section{SPECIMEN HANDLING}

Blood was collected into $7 \mathrm{ml}$ vacutainer serum separator tubes and allowed to clot for $2 \mathrm{hr}$ at ambient temperature. Serum was separated from clotted blood by low-speed centrifugation for 15 $\min$. The serum was transferred into fresh tubes, frozen on dry ice, shipped to the laboratory in Bangkok, Thailand, and stored at $-20^{\circ} \mathrm{C}$.

\section{Determination of Serum Enzyme Levels}

Serum activities of alanine aminotransferase (ALT), asparate aminotransferase (AST), and total bilirubin were neasured by kinetic spectroscopy using a Clinical System 700 analyzer (Beckman Instruments, Palo Alto, $\mathrm{CA}$ ) with reagents and standards from the same, manufacturer. 


\section{Serologic Assays}

Sera were examined for IgM to HAV, for IgM to hepatitis B core antigen (HBcAG), for hepatitis $B$ surface antigen ( $\mathrm{HBsAg}$ ), and for total immunoglobulin to hepatitis $\mathrm{C}$ virus (HCV) commercial enzyme-linked immunosorbent assay (EISSA) test kits (HAVABO - using Corzyme (B)-M, AUSYMEB Monocolnal, and HCV North Chicago, IL). Assays were performed as recommended by the manufacturer

Sera were examined for IgG and IgM to HEV using commercially available ELISA tests (Diagnostic Biotechnology Co., Ltd., Singapore). These tests employ three recombinant HEV fusion proteins derived from expression of strongly antigenic portions of the putative structural proteins of both HEV Burma B and Maxico, 1986 isolates [Yarbough et al., 1991]. Assays were performed and analyzed as recommended by the manufacturer and as previously reported [Clayson et al., 1995c]. when conducted in this manner, the assay is highly specific (specificity score $=0.96$ ) and moderately sensitive (sensitivity score $=9.82$ ) for detecting antibody responses elicited by acute infection [Clayson et al., 1995c]. The sensitivity of the test to detect antibody years after infection is unclear.

\section{Polymerase Chain Reaction}

RNA was extracted from serum with silicon dioxide as described [Clayson et al., 1995c]. Olgonucleotides, cDNA synthesis, nested DNA amplification, detection of amplified DNA, and contamination control procedures for this study were as described [Clayson et al., 1995b]. Primers for PCR were based on sequences from the putative RNA polymerase gene region of open reading frame 1 of the Burma isolate of HEV [Tam et al., 1991]. Amplified DNA fragments were confirmed as HEV sequences by hybridization with an oligonucleotide probe specific for HEV [Clayson et al., 1995b].

\section{Data Collection}

For each soldier, the date of disease onset, age, date of arrival at the training camp, and previous duty location were provided by Nepalese military medical officials from military records.

TABLE I.

Characteristics of 32 Hepatitis Patients at a Military Training Camp in Nepal

\begin{tabular}{ll}
\hline Characteristic & Value \\
\hline Age, median years (range) & $29(22-38)$ \\
Initial signs and symptoms (\%) & \\
Scleral interus & 100 \\
Jaundice & 97 \\
Dark Urine & 94 \\
Anorexia & 69 \\
Liver tenderness & 56 \\
Malaise & 47 \\
Nausea & 28 \\
Serum ALT levels, median U/L & $1,124(40-4,240)$ \\
(range) $^{\mathrm{a}}$ Sernum total bilirubin levels, median & $97(22-438)$ \\
Umol/L (range) & \\
\hline
\end{tabular}

Clayson et al.

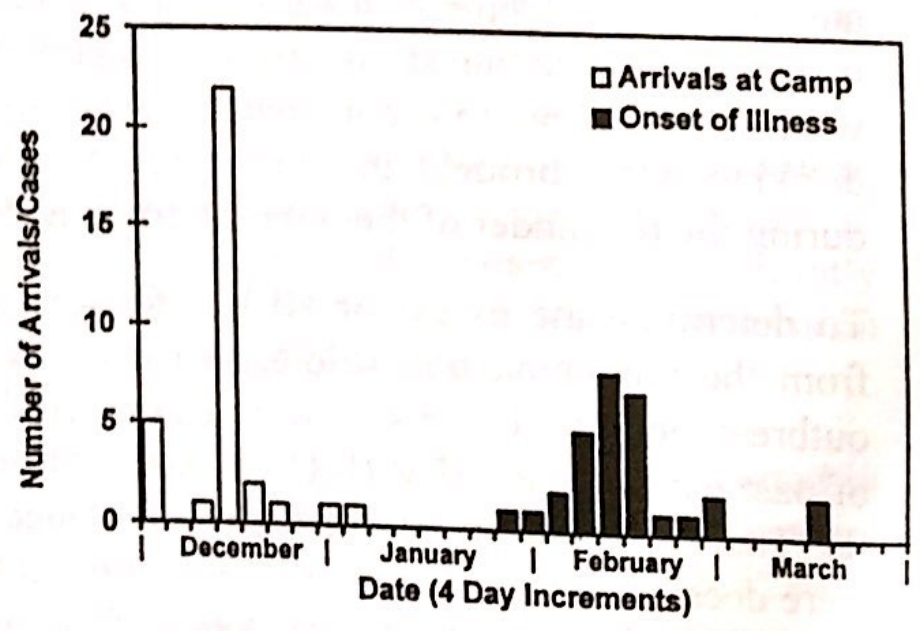

Fig. 1. Distribution of arrivals and onset of illness among 32 hepatitis cases at a military training camp in Nepal. Nepalese soldiers arrived at the camp to form and train a new unit for deployment. Approximately 8 weeks after arrival, 32 soldiers became ill with hepa tients (white bars) as distributions and dates of arrival of the 32 pabars). 


\section{RESULTS}

Approximately 8 weeks after 692 soldiers arrived at the training camp, the loc physician noted several cases of acute the soldiers. All patients had an abrupt onset In all, cases of acute hepaticed with jaundiced with associated scleral icterus and dark urine of $1 \mathrm{~h}_{\mathrm{he}}$ were done in Bangkok on frozen sera 3-10 days after collection; therefore, the measured $\mathrm{Al}$,
levels may be artificially low, accounting for normal or minimally elevated determination resume normal duties within 4 weeks.

To determine the etiology of the hepatitis cases, patient sera were examined for evidence infection with $\mathrm{HAV}, \mathrm{HBV}, \mathrm{HCV}$, and HEV. all patients had evidence of immunity to $\mathrm{HAV}$ all had IgG but none had IgM to HAv. Neither HBV surface antigen nor IgM to HBV core antigen was detected in any of the patients. In additon, total immunoglobulin to $\mathrm{HCV}_{\text {was }} \mathrm{w}_{\mathrm{O}}$ detected in 29 of 32 patients. However, IgM to HeV was detected in 29 of 32 patients and HEv RNA was detected in 29 of 32 patients and HEV RNA was detected in the sera of 25 of 32 patients. In all, acute markers of HEV infection were detected in 30 of the 32 patients implicating HEV as the responsible etiologic agent.

We considered the possibility that multiple sources of infection may have been responsible for the cases of hepatitis observed. However, several factors indicated that a common source was responsible for the outbreak and that the source was located at the camp. First, the distribution of hepatitis cases over time was consistent with the distribution expected in a focal outbreak and was not consistent with random sporadic transmission (Fig. 1). Second, the 8 weet average period between the soldiers arrival at the camp and the onset of illness suggested that soldiers were exposed to HEV at the camp. In addition, the wide geographical dispersion of the 32 hepatitis E patients just prior to their arrival at the camp (Fig. 2) makes infection prior to arrival at the camp seem unlikely. The source of infection was not definitively identified but fecally contaminated drinking water was suspected. Water source used by the soldiers consisted of springs and creeks that were accessible to potential contamination from people or animals. In fact, these natural water sources were located nearby and downhill from inadequately maintained pit latrines. To prevent the further spread of HEV infections, soldiers were instructed not to use the natural water sources and were provided with containers of safe drinking water brought in from a nearby town. No further Hepatitis cases were observed during the remainder of the 6 month training period.

To determine the extent of HEV infections among soldiers without clinical hepatitis, sera from the remaining 660 soldiers were collected in early March 1995 (near the end of the outbreak period). These sera were examined for antibodies to HEV and HEV RNA Evidence of past exposure to HEV (IgG but not IgM nor HEv RNA was found in 204 soldiers (Table II). Therefore, the initial antibody prevalence was estimated to be $30 \%$ and 488 individuals were deemed susceptible to HEV infection at the outset of the outbreak. Assuming that all 32 patients had hepatitis E, the incidence of disease was found to be approximately $7 \%$ (32/488) Acute markers for HEV infection (IgM to HEV and/or HEv RNA were found in an additional 83 soldiers (Table II). Combining these 83 soldiers with the 32 patients gives an overall

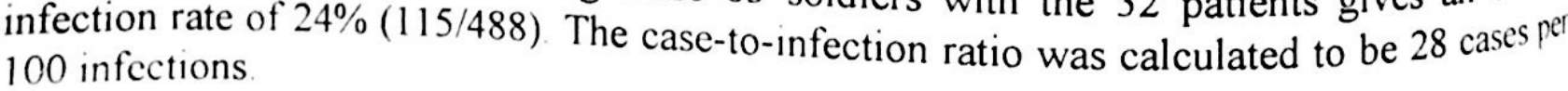




\section{DISCUSSION}

Our results indicate that the agent responsible for an outbreak of acute hepatitis among Nepalese soldiers was HEV. No. Marker of recent HAV, HBV, or HCV infection were detected in any of the patients. In fact, all soldiers were found to be immene to HIV as IgG but not IgM to HIV was detected in all soldiers. This observation is consistent with previous findings that virtually all Nepalese are immune to HAV by 5 years of age [Shrestha, 1986; Clayson et al., 1995a] However, markers of recent or current HEV infection weere detected in 30 of the 32 patients. The observed signs and symptoms among the hepatitis patients in this study were similar to those previously observed in hepatitis E patients in other studies [Zahuang et al, 1991; Tsega et al; 1991; Clyson et al., 1995c]. And finally, the detection of IgM to HEV and of HEV RNA in the vast majority of patients in this study is also consistent with the detection of these markers in hepatitis $\mathrm{E}$ patients in previous studies [Nanda et al., 1995; Clayson et al., 1995c].

We were unable to find in the literature estimates of infection rates, disease rates, or case-toinfection ratios for conditions of epidemic HEV transmission in NEPAL. The occurrence of an outbreak of hepatitis $\mathrm{E}$ in an isolated area with a well-defined population provided a unique opportunity to characterize the epidemiology of an outbreak of hepatitis E. Information regarding infection rates, disease rates, and case-to-infection ratios under conditions of sporadic virus transmission in Nepal has recently become available [Clayson et al., 1997]. Therefore, this outbreak also provided an opportunity of compare the epidemiology of HEV during conditions of epidemicvs. Sopradic virus transmission.

We found evidence of past exposure to HEV (IgG in the absence of both IgM and HEV RNA) in approximately $30 \%$ of all soldiers at the camp (ages 20-40). This figure is nearly identical to the antibody precvalence previously reported for this age group in Nepal [Longer et al., 1994; Clayson et al., 1997]. The disease and infection rates observed during this outbreak ( $7 \%$ and sporadic virus transmission ( $2.8 \%$ and $9.2 \%$, respectively) [Clayson et al., 1997]. This result indicates that a higher proportion of soldiers were exposed to the virus during this outbreak than would be exposed during conditions of sporadic transmission.

If people are exposed to significantly higher levels of virus during conditions of epidemic virus transmission than they are during sporadic transmission, then one would expect that the case-toinfection ratio would be higher during epidemic transmission because exposure to higher levels of virus would be expected to more easily overcome the intrinsic host defenses. However, the caseto-infection ratio during this out break (28 per 100 infections) was nearly identical to the case-toinfection ratio observed in a similar age group under sporadic conditions ( 30 per 100 infections). This result suggests either that during this outbreak soldiers were not exposed to significantly higher levels of virus than are people in the same age group in Nepal during sporadic transmission or that virus dose is not a determinant of whether illness will result from infection.

This outbreak occurred in military camp. Similar outbreaks of hepatitis $\mathrm{E}$ have been reported in other military environments [Sanyal, 1957; Joshi et al., 1984; Zhuang et al., 1991]. An average duration of confinement due to illness of 3 weeks was reported during one outbreak in Ethiopia where 423 cases were reported among soldiers. A similar length of hospital stay was observed in the present study as well as in others [Clayson et al., 1995c]. High attach rates and lengthy convalescent periods can lead to significant loss of soldier duty days. Therefore, HEV can have a serious impact on military operations in HEV endemic areas under conditions where sanitation and personal hygiene have broken down, or where safe drinking water is unavailable. 